\title{
« Nous et vous au cœur de l'Europe »: la relation austro-tchécoslovaque durant les années 1960 vue de Prague
}

Hélène Leclerc, "Wir und Sie im Herzen Europas »: die österreichischtschechoslowakische Beziehung in den 1960er Jahren von Prag aus gesehen Hélène Leclerc, "Wir und Sie im Herzen Europas": the Austrian-Czechoslovak relation during the 1960's regarded from Prague

Hélène Leclerc

\section{OpenEdition}

Journals

Édition électronique

URL : https://journals.openedition.org/austriaca/857

DOI : 10.4000/austriaca.857

ISSN : 2729-0603

Éditeur

Presses universitaires de Rouen et du Havre

Édition imprimée

Date de publication : 1 décembre 2019

Pagination : 149-162

ISBN : 979-10-240-1454-8

ISSN : 0396-4590

\section{Référence électronique}

Hélène Leclerc, « « Nous et vous au cœur de l'Europe » : la relation austro-tchécoslovaque durant les années 1960 vue de Prague », Austriaca [En ligne], 88-89 | 2019, mis en ligne le 31 décembre 2020 consulté le 24 octobre 2022. URL : http://journals.openedition.org/austriaca/857 ; DOI : https:// doi.org/10.4000/austriaca.857 
Hélène LECLERC

Université Toulouse-Jean-Jaurès,

Centre de recherches et d'études germaniques (CREG, EA 4151)

\section{«Nous et vous au cour de l'Europe»: la relation austro-tchécoslovaque durant les années 1960 vue de Prague}

En janvier 1958 paraissait à Prague une nouvelle revue, éditée en langue allemande et destinée aux lecteurs germanophones de l'étranger, principalement de République fédérale d'Allemagne. Cette revue, intitulée Im Herzen Europas. Tchechoslowakische Monatsschrift ${ }^{1}$, en laquelle d'aucuns ont pu voir «le magazine le plus intéressant du bloc de l'Est ${ }^{2}$ » et un porte-voix officiel du ministère des Affaires étrangères tchécoslovaque ${ }^{3}$, était conçue comme un magazine culturel visant à promouvoir le patrimoine et la culture tchécoslovaques en Allemagne de l'Ouest, mais aussi en Autriche, Suisse et au Luxembourg, ainsi que, quoique plus modestement, en République démocratique allemande. On s'y intéressait à la politique, à l'histoire, à la littérature, à la philosophie, aux sciences (humaines, naturelles, physiques ou médecine), au

1. Im Herzen Europas. Tschechoslowakische Monatsschrift, Prag, Orbis, 1958-1971.

2. Bernd-Lutz Lange, "Im Herzen Europas», dans Bernd-Lutz Lange, Mauer, Jeans und Prager Frühling, 2003, Berlin, Aufbau-Verlag, 2012, p. 283.

3. J. Herda, G. Pacurar, «Hier spricht man nicht nur Deutsch: Deutsch- und anderssprachige Medien in der Tschechischen Republik», dans Walter Koschmal, Marek Nekula et Joachim Rogall (dir.), Deutsche und Tschechen. Geschichte. Kultur. Politik, München, Beck, 2001, p. 377-386, ici p. 378. La maison dédition Orbis avait vu le jour en mars 1921 dans le but de répondre au projet du ministère des Affaires étrangères tchécoslovaque, dirigé alors par Edvard Beneš, de disposer d'une institution capable d'informer sur la vie en Tchécoslovaquie et de contribuer à la diffusion de cette information à l'étranger. Des liens étroits avec l'État et la politique du gouvernement demeurèrent la caractéristique de la maison d'édition en dépit des changements de régime. Zdeněk Šimeček souligne la dimension de propagande au service du régime tchécoslovaque après 1945, en présentant Orbis comme «une institution du ministère de l'Information fabriquant avant tout des ouvrages de propagande destinés à l'étranger» (Zdeněk Šimeček, Geschichte des Buchhandels in Tschechien und in der Slowakei, Armin Hetzer [trad.], Wiesbaden, Harrassowitz, 2002, p. 184). Sur Orbis, voir également : Michal Přibáň et al., Česká literární nakladatelství 1949-1989 (Les maisons d'édition littéraires tchèques), Praha, Academia, 2014, p. 345-380, et Jan Halada, Encyklopedie českých nakladatelství 1949-2006 (Encyclopédie des maisons dédition tchèques 1949-2006), Praha, Libri, 2007, p. 249-250. 
théâtre, au cinéma, aux arts plastiques, à la photographie, ou encore au sport, le tout étant agrémenté de nombreuses illustrations et photographies. À travers cette diffusion de la culture tchécoslovaque, il s'agissait aussi d'œuvrer à amorcer, puis à intensifier les relations tchéco-allemandes, si bien qu'on peut considérer Im Herzen Europas comme un instrument de diplomatie culturelle du régime tchécoslovaque, d'autant plus intéressant que le mensuel s'insère dans le contexte du processus de libéralisation du régime tchécoslovaque des années 1960 et se fait l'écho enthousiaste des réformes qui se mettent en place à partir de janvier 1968. Un grand nombre des acteurs de la vie intellectuelle et culturelle tchécoslovaque, qui connaît dans les années 1960 un extraordinaire épanouissement, y collaborèrent ${ }^{4}$ ou bien y virent leur œuvre présentée $e^{5}$ À la suite de l'échec et de la répression de la tentative d'instaurer un "socialisme à visage humain» en Tchécoslovaquie lors du «Printemps de Prague» en 1968, plusieurs collaborateurs de la revue furent interdits de publication, à l'exemple de celle qui en fut d'abord la rédactrice en chef adjointe, Lenka Reinerová ${ }^{6}$, et la revue cessa finalement de paraitre sous cette forme en 1971.

L'objet du présent article n'est pas la revue Im Herzen Europas ${ }^{7}$, mais sa "petite sœur», Wir und Sie im Herzen Europas (WuS), version spécifiquement destinée aux lecteurs autrichiens, publiée d'août 1961 à mai $1970^{8}$. Cette déclinaison autrichienne lancée plus de trois ans et demi

4. On peut citer entre autres l'écrivain et dramaturge Pavel Kohout, les artistes Karel Trinkewitz et Adolf Hoffmeister, le germaniste Eduard Goldstücker, ou encore les photographes Václav Jirů et K. O. Hrubý.

5. Václav Havel, Milan Kundera, Miloš Forman, pour ne citer que quelques noms parmi les plus célèbres, ou encore le poète Jiř́i Gruša, qui écrit alors en tchèque et dont les poèmes sont traduits par Peter Pont (alias Oskar Kosta). Sur Gruša, voir l'article de Renata Cornejo dans ce même numéro d'Austriaca.

6. La journaliste et écrivain de langue allemande Lenka Reinerová (1916-2008) fut rédactrice en chef adjointe de la revue de 1958 à octobre 1968, puis, après le départ du rédacteur en chef Gustav Solar, rédactrice en chef de novembre 1968 à décembre 1969, avant dêtre à son tour renvoyée en 1970, exclue du Parti communiste et frappée d'interdiction de publier et décrire. Elle travailla ensuite comme interprète et traductrice, mais de façon anonyme, et écrivit «pour son tiroir». Ses premiers récits et nouvelles parurent en 1983 chez Aufbau à Leipzig.

7. Nous avons consacré à la revue Im Herzen Europas l'inédit de notre dossier d'habilitation à diriger des recherches (Université Toulouse-Jean Jaurès, 2019). La publication en est prévue.

8. L'arrêt de la publication est concomitant du départ de Lenka Reinerová de la rédaction de Im Herzen Europas, qui signifie la fin de la revue telle quelle était conçue jusque-là. Celle-ci subsiste certes, mais sous un autre titre (Tschechoslowakisches Leben), et dans une version «normalisée» qui n’a plus grand-chose à voir avec celle qu’anima Lenka Reinerová pendant douze ans. 
après son aînée et éditée à environ 4000 exemplaires $^{9}$ se propose de mettre l'accent sur les relations austro-tchécoslovaques, dans un esprit de médiation et de rapprochement, comme l'annonce le numéro de juillet 1961 de Im Herzen Europas :

Eine jüngere Schwester unserer Zeitschrift tritt an die Stelle der älteren, um uns und Sie, Menschen in der Tschechoslowakei und in Österreich, die beide im Herzen Europas liegen und so vieles Gemeinsame haben, näher zusammenführen und über die Unterschiede hinweg, die es zwischen unseren Ländern geben mag, eine gemeinsame Sprache finden zu lassen.

Wir wollten das schon im Titel zum Ausdruck bringen. Darum heißt die neue Zeitschrift WIR und SIE IM HERZEN EUROPAS.

Sie hat es sich zum Ziel gesetzt, Ihnen ein Fenster ins nördliche Nachbarland Österreichs ganz weit zu öffnen, Sie über alle Reisemöglichkeiten und -erleichterungen zu informieren, Ihnen die Schönheit seiner Landschaft, seine Kunstdenkmäler und das Leben ihrer Menschen vor Augen zu führen, gemeinsame Kulturtraditionen zu pflegen und die wirtschaftlichen Berührungspunkte aufzuzeigen. Das mag anspruchsvoll klingen, aber wir werden uns nicht weniger intensiv auch Sportfragen widmen, wir wollen Seiten für die Frauen mit Moden und Kochrezepten bringen, neue Briefmarken zeigen, die letzten Witze erzählen und vieles andere mehr ${ }^{10}$.

Le premier numéro de WuS proclame quant à lui : "Damit wir und Sie im Herzen Europas als Nachbarn nebeneinander besser verstehen, wird diese Zeitschrift herausgegeben ${ }^{11}$.» Les objectifs et la méthode sont donc affichés : une rhétorique qui cultive l'amitié, met en avant la proximité géographique, culturelle et historique et entend souligner la spécificité de la relation austro-tchécoslovaque et la promouvoir. «Nous et vous au cœur de l'Europe», c'est affirmer une appartenance commune des deux États, une position géographique particulière à la jonction des blocs occidental et oriental, et partant, peut-être une mission européenne commune, tout en se positionnant comme initiateur («nous», puis «vous») ; c'est aussi, par le biais de cette relation privilégiée qu'on postule avec l'Autriche, une façon de contester à l'Allemagne la position prééminente quelle revendiquerait «au cœur de l'Europe ${ }^{12} »$. Le nom

9. Avec Im Herzen Europas, c'est un total d'environ 12000 exemplaires par mois diffusés dans les pays germanophones.

10. Im Herzen Europas, juillet 1961, [s. p.]. Les passages en romains et en lettres capitales correspondent au texte original.

11. WuS, août 1961, [s. p.].

12. Renata Fritsch-Bournazel attribue à $\mathrm{M}^{\mathrm{me}}$ de Staël l'origine de cette métaphore associée à l'Allemagne et montre combien la description d'une Allemagne située au centre de 
même de la revue révèle une relation perçue du côté tchécoslovaque comme emblématique de la possibilité pour deux États obéissant à des systèmes de société différents de cultiver des espaces de rencontre ${ }^{13}$.

Sur la forme, les deux revues sont strictement identiques (même format, même mise en page, même couverture). Concernant le contenu, on relève un grand nombre d'articles communs aux deux éditions, avec en moyenne entre trois et cinq articles spécifiquement rédigés chaque mois pour la version autrichienne ; il arrive toutefois qu'un article paru dans WuS paraisse le mois suivant dans Im Herzen Europas et vice versa. La direction de la rédaction est la même : Gustav Solar ${ }^{14}$ est rédacteur en chef, Lenka Reinerová rédactrice en chef adjointe, leur sont associés le journaliste et photographe Milan Škarýd ${ }^{15}$ et l'artiste Karel Trinkewitz $^{16}$, qui apparaissent dans l'ours comme rédacteurs à partir de

l'Europe parcourt l'histoire comme une constante. Voir Renata Fritsch-Bournazel, Europa und die deutsche Einheit, Bonn, Aktuell, 1991, p. 171. Ce qualificatif lui est cependant disputé à la fois par la Pologne et par les Pays tchèques, puis la Tchécoslovaquie, dont le premier président et fondateur, Masaryk, considérait que l'Europe centrale regroupait toutes les petites nations d'Europe, mais surtout pas l'Allemagne et l'Autriche (voir Timothy Garton Ash, La chaudière. Europe centrale, 1980-1990, Paris, Gallimard, 1990, p. 195) et s'opposait à la conception de la Mitteleuropa de l'Allemand Friedrich Naumann (voir Steffen Höhne, «Imperiale Ambitionen und das Recht der kleinen Nationen. „Mitteleuropa“ bei Naumann und Masaryk», dans Jacques Lajarrige, Walter Schmitz et Giusi Zanasi dir., Mitteleuropa. Geschichte eines transnationalen Diskurses im 20. Jahrhundert, vol. 1, Dresden, Thelem, 2011, p. 143-168).

13. C'est aussi ce que souligne le vice-ministre des Affaires étrangères tchécoslovaque Otto Klička lors d'une cérémonie de la Société austro-tchécoslovaque à Vienne le 10 mai 1965. Voir Karl Peterlik, «Komplexe Beziehungen. ČSSR», dans Oliver Rathkolb, Otto M. Maschke et Stefan August Lütgenau (dir.), Mit anderen Augen gesehen. Internationale Perzeptionen Österreichs 1955-1990. Österreichische Nationalgeschichte nach 1945, Bd. 2, Wien et al., Böhlau, 2002, p. 621.

14. La biographie de Gustav Solar n'est pas aisée à reconstituer ; on trouve quelques éléments à son sujet dans Jindřich Dejmek, Diplomacie Československa, Díl II. Biografický slovník československých diplomatů (1918-1992), Praha, 2013, p. 580 (http://www.mzv.cz/jnp/ cz/o_ministerstvu/organizacni_struktura/utvary_mzv/specializovany_archiv_mzv/ kdo_byl_kdo/solar_puvodne_schwarzkopf_gustav.html). De son vrai nom Schwarzkopf, Solar est né en 1916 dans une famille pragoise juive et germanophone. Déporté à Terezín et Auschwitz, il entre dès avril 1945 au ministère des Affaires étrangères tchécoslovaque, où son statut de membre du parti communiste lui permet de devenir à partir de 1948 chef de la section ouest-européenne du département de politique. Il doit quitter le ministère en 1957, «en raison de ses origines» selon Jindřich Dejmek, et il rejoint la radio pragoise. Jindřich Dejmek ne mentionne nullement son activité au sein de Im Herzen Europas. Après la répression du Printemps de Prague, il émigre en Suisse. Il signe son dernier éditorial pour Im Herzen Europas en octobre 1968.

15. Né en 1930, ce photographe est l'un des principaux collaborateurs des deux revues.

16. Karel Trinkewitz (1931-2014) est un auteur et artiste (collagiste, peintre, dessinateur, 
décembre 1964. Cette équipe s’adjoint en outre, à partir de 1962, les services d'une correspondante spéciale à Vienne, en la personne de Gerda Rothmayer ${ }^{17}$. Le réseau personnel viennois de Lenka Reinerová joue ici un rôle important puisque Gerda Rothmayer est l'épouse du journaliste autrichien Bruno Frei (1897-1988) avec lequel la journaliste pragoise entretient de solides liens d'amitié depuis leurs années d'exil au Mexi$q^{q u}{ }^{18}$. L'atteste en particulier une correspondance régulière entre Lenka Reinerová, Bruno Frei et Gerda Rothmayer échangée jusqu’au décès de $\mathrm{Frei}^{19}$. On y apprend entre autres que Lenka Reinerová avait assez régulièrement l'occasion de se rendre à Vienne et que ce cercle d'amis comptait également l'intellectuel communiste autrichien Ernst Fischer (1899-1972). Leurs échanges épistolaires concernent pour une part relativement importante leurs activités journalistiques et leurs revues respectives et Bruno Frei sollicite son amie, dans une lettre du $1^{\text {er }}$ avril 1960, pour la tenue d'une correspondance pragoise destinée à la revue Tagebuch dont il vient de reprendre la direction, proposition quaccepte Lenka Reinerová, anticipant ainsi la mission future de $W u S$ visant à faire connaître la Tchécoslovaquie en Autriche.

caricaturiste et illustrateur de livres) de langues allemande et tchèque; il collabore à Im Herzen Europas à partir de juin 1961. Sur cet artiste, voir notamment : Karel Trinkewitz, «Meine kurze Autobiographie», dans Christine Gölz, Alfrun Kliems et Birgit Krehl (dir.), „Die unerträgliche Leichtigkeit des Haiku“. Der Künstler Karel Trinkewitz, WettinLöbejün, Janos Stekovics, 2016, p. 11, et Jürgen Herda, «Kapitalismus mit Fratze. Karel Trinkewitz: Zum 80. Geburtstag des Künstlers und Charta-77-Unterzeichners », Europe online magazine, 17 décembre 2011, http://www.europeonline-magazine.eu/ kapitalismus-mit-fratze_161856.html (consulté le 2 septembre 2018).

17. D’après Karl Peterlik (art. cité, p. 630), Gerda Rothmayer écrivait également pour le Prager Volkszeitung (anciennement Aufbau und Frieden), organe de presse de la minorité germanophone de Tchécoslovaquie, auquel collaboraient de nombreux journalistes de la revue Im Herzen Europas, à commencer par Lenka Reinerová elle-même. Sur ce journal destiné à la minorité allemande de Tchécoslovaquie, voir notamment : Manuela Olhausen, Politische Kommunikation im Wandel. Die deutschsprachige Presse des (ehemaligen) Ostblocks zwischen 1980 und 2000. Eine Inhaltsanalyse der Zeitungen Neue Zeitung, Ungarn, Prager Volkszeitung, Tschechoslowakei/Tschechien, Neuer Weg/ Allgemeine Deutsche Zeitung für Rumänien, Rumänien, Neues Leben, Sowjetunion/ Russland in den Jahren 1980, 1989, 1990 und 2000, Hamburg, Dr. Kovač, 2005, et Lukáš Novotný, "Deutsche Minderheit und ihre Presse», Aussiger Beiträge. Germanistische Schriftenreihe aus Forschung und Lehre, ${ }^{\circ} 10,2016$, p. 145-156.

18. Lenka Reinerová a sans doute fait la connaissance de Bruno Frei dès 1933 alors qu'il était en exil à Prague ; Frei fut du reste rédacteur en chef de l'hebdomadaire antifasciste Der Gegenangriff, dont Lenka Reinerová assura elle aussi un temps la rédaction.

19. Quelques lettres de cette correspondance sont conservées aux archives littéraires de l’Académie des Arts de Berlin (fonds «Lenka Reinerová», en attente de classement). 
La parution de $W u S$ s'inscrit dans un contexte de détente des relations bilatérales favorisé par le contexte international. Après un nouveau départ difficile entre 1945 et 1955, où la situation internationale déterminait fortement la qualité de la relation austro-tchécoslovaque ${ }^{20}$, les années 1960, du fait de la déstalinisation amorcée dans le bloc communiste $^{21}$, instaurent des conditions plus propices à un rapprochement entre ces deux États qui, rappelons-le, ne disposent jusqu’en 1975 que d'une simple représentation diplomatique ${ }^{22}$. Du côté autrichien, on commence à s'intéresser davantage à ses voisins, en particulier à la Tchécoslovaquie, et ce au sein des deux grands partis autrichiens, d'abord l'ÖVP du chancelier Klaus qui fait de l'Ostpolitik un pilier de la politique étrangère autrichienne, puis le SPÖ sous la présidence de Bruno Kreisky, qui voit dans l'espace danubien la mission centrale de la politique étrangère autrichienne ${ }^{23}$. Côté tchécoslovaque se manifeste une volonté de développer les échanges ; l'émissaire autrichien Calice note dès 1961 une évolution des esprits à Prague, désormais marquée par le souhait de montrer que la Tchécoslovaquie est un pays culturellement orienté à l'ouest ${ }^{24}$. Cette évolution se traduit d'abord par un assouplissement des contraintes concernant les voyages. Si l'Autriche persiste à refuser toute institutionnalisation des échanges culturels ${ }^{25}$, un pas est néanmoins franchi en septembre 1964 avec l'inauguration des «Stadtgespräche Prag-Wien», émission conjointement réalisée par la télévision autrichienne (ORF) et la télévision tchécoslovaque et diffusée en direct et en traduction simultanée ${ }^{26}$. L'esprit de ces conver-

20. Miroslav Šepták, «Die Zweite Republik: Vom Ende des Zweiten Weltkriegs bis zum Staatsvertrag. Ein Abriss der tschechoslowakisch-österreichischen politischen Beziehungen 1945-1955 », dans Miroslav Kunštát, Jaroslav Šebek et Hildegard Schmoller (dir.), Krise, Krieg und Neuanfang. Österreich und die Tschechoslowakei in den Jahren 1933-1948, Berlin, Lit, "Schriftenreihe der Ständigen Konferenz österreichischer und tschechischer Historiker zum gemeinsamen kulturellen Erbe, 2 », 2017, p. 238-239.

21. Karl Peterlik, «Komplexe Beziehungen. ČSSR», art. cité, p. 618.

22. Des ambassades ne seront ouvertes qu'en 1975 après la signature de l'accord sur les biens autrichiens.

23. Voir Alexander Burka, Was blieb vom Fenster in den Westen? Zur Auslandspolitik Österreichs in Ostmitteleuropa seit 1945 am Beispiel Polens und der Tschechoslowakei/ Tschechiens, Francfort et al., Peter Lang, 2012, p. 135.

24. Ibid., p. 193.

25. Ibid.

26. Voir le récit qu’en fait Helmut Zilk (1927-2008), alors présentateur autrichien de l'émission : «Zum Beginn des „Prager Frühlings": die „Stadtgespräche Prag-Wien“», dans Stefan Karner, Natalja Tomilina, Alexander Tschubarjan et al. (dir.), Prager Frühling. Das internationale Krisenjahr 1968, Köln et al., Böhlau, 2008, 2 vol., p. 1089-1093. Il faut 
sations bilatérales télévisuelles rejoint tout à fait la mission que s'est assignée $W u S$, qui se propose d'emblée de creuser le sillon amorcé par l'émission :

[...] Wir wollen versuchen, in den nächsten Nummern unserer Zeitschrift einige dieser brennend wichtigen Fragen näher zu beleuchten. Vom tschechoslowakischen und österreichischen Standpunkt aus. [...]

Worte, die uns einander näherbringen können, sind willkommen. Denn solchen Worten folgen gute Taten ${ }^{27}$.

Dès son lancement en 1961, WuS s'est en effet fixé deux objectifs principaux : 1) faire connaître la Tchécoslovaquie en Autriche et réviser son image négative ; 2 ) amorcer des relations culturelles. Comme l’a montré Paul Ullmann, l'image de la Tchécoslovaquie en Autriche, et particulièrement dans ses médias, est en effet, jusqu’au début des années 1960, fortement négative : "Man ging davon aus, dass sich in diesem Lande ein totalitäres stalinistisches Regime etabliert hatte ${ }^{28}$.»WuS souligne régulièrement l'ampleur des préjugés négatifs à l'égard de la Tchécoslovaquie qui auraient cours en Autriche ${ }^{29}$, pour souvent s'en amuser avec une ironie mordante. C'est par exemple le cas d'un article consacré par Karel Trinkewitz à la visite à Prague d'un couple d'Autrichiens lauréats du concours des lecteurs de WuS, occasion pour son auteur d'une attaque en règle contre la prétendue "presse libre» autrichienne ${ }^{30}$. M. et $\mathrm{M}^{\mathrm{me}}$ Stauder, de Bleiberg bei Villach, sont ainsi, à la manière des deux voyageurs persans de Montesquieu, invités à sétonner de ce qu'ils

désormais cependant considérer avec précaution, comme le fait Alexander Burka (Was blieb vom Fenster in den Westen?, op. cit., p. 193), le témoignage de Helmut Zilk, dont ont été révélées en 2009 les activités d'informateur des services secrets tchécoslovaques. À propos de cette émission, signe manifeste d'une libéralisation du régime tchécoslovaque, Karl Peterlik souligne que la "coexistence pacifique», telle qu'elle put y être pratiquée, ne signifia pas pour autant que l'on renonçât à toute confrontation idéologique («Komplexe Beziehungen. ČSSR», art. cité, p. 619); Alexander Burka parle quant à lui de «communication comme confrontation» (op. cit., p. 183).

27. Lenka Reinerová, «I. Stadtgespräch Prag-Wien und was daraus werden sollte», WuS, novembre 1964, p. 23.

28. Paul Ullmann, Eine schwierige Nachbarschaft. Die Geschichte der diplomatischen Beziehungen zwischen Österreich und der Tschechoslowakei von 1945-1968, Wien, Lit, 2006, p. 215.

29. Les Tchèques et les Slovaques sont du reste mal informés eux aussi sur l'Autriche, comme le révèle l'article de Karel J. Matouš «Liegt Österreich in Australien ?» publié en mai 1968. Voir Karl Peterlik, «Komplexe Beziehungen. ČSSR», art. cité, p. 627.

30. Karel Trinkewitz, „Fünf Tage in Prag verbrachte das Ehepaar Stauder aus Bleiberg als Gewinner unseres vorjährigen Wettbewerbs", WuS, septembre 1962, p. 20-21. 
découvrent lors de leur séjour, non toutefois pour s'en moquer comme les protagonistes des Lettres persanes, mais au contraire pour dénoncer le mensonge dans lequel l'Autriche entretiendrait sa population et les défaillances - matérielles et morales - du système capitaliste :

„Die Menschen sind hier ebenso geschmackvoll angezogen wie bei uns. Aber die Preise in den Schaufenstern der Kleidergeschäfte sind meist niedriger als bei uns." [...] Auf dem Gehsteig steht ein Stuhl mit einem Packen Zeitungen und einem Kästchen voll Kleingeld. „Selbstbedienung? Bei uns hätten wir Angst, daß wir nach einer Weile nicht einmal den Stuhl mehr finden. "Alle paar Schritt gehen wir an einem Buchladen vorbei. „Auflagen von 35 000? Und daß die Leute vor der Buchhandlung Schlange stehen? Nein, das gibt's bei uns auch nicht." Und die Prager Kirchen: „So viele Türme! Die Instandhaltung und Restaurierung zahlt der Staat? Das wird uns keiner glauben." Doch auch bei uns in der Tschechoslowakei gibt es gewisse Dinge nicht. „Bei euch gibt's keine Bettler? Und auch keine Arbeitslosen?" Nein. Und auch keine Ärztestreiks, kein Arzt lehnt ab, kranke Arbeiter zu behandeln. [...] Lidice. [...] „Führt ihr eure Kinder hierher, damit sie nicht vergessen? Unseren Kindern sagt keiner, was Faschismus ist"...

Un an plus tard, reprenant les mêmes accusations à l'encontre de la presse viennoise, Rudolf Jelinek rapporte au retour d'un séjour à Vienne, où sa tante, persuadée qu'on mourait de faim en Tchécoslovaquie, lui aurait remis un colis de provisions : "Was man doch alles über seine Heimat erfahren kann! [...] Wohl sind wir Nachbarn, aber mir schien es, daß viele Bewohner Wiens von Prag und der ČSSR weniger wissen als über die USA, und das wenige ist verzerrt ${ }^{32}$."

Ces préjugés à l'égard des conditions de vie en Tchécoslovaquie sont tenaces, l'atteste un nouveau démenti publié en juillet 1965, émanant cette fois d'un touriste viennois. WuS sollicite en effet désormais les témoignages de visiteurs de la Tchécoslovaquie, quelle promet de publier «même s'ils ne représentent pas toujours l'opinion de la rédaction", ce qui se révèle un moyen bien plus efficace pour corriger clichés et idées fausses. Quel meilleur avocat pour le régime tchécoslovaque que ce touriste autrichien dénonçant les mensonges qu’on lui conterait dans son pays :

31. Ibid. La rédaction recourt ici à une variante de la méthode Andere über uns, qui consiste à convier des journalistes étrangers pour qu'ils rapportent ensuite des faits positifs sur la Tchécoslovaquie, età la méthode Sie über sich, oùl'on met en évidenceles descriptions négatives d’Autrichiens sur leur propre pays. Ces deux méthodes étaient notamment préconisées et utilisées par l'agence Panorama de RDA, chargée de l'information à destination de l'étranger. Voir Thomas Brünner, Public Diplomacy im Westen. Die Presseagentur Panorama DDR informiert das Ausland, Francfort et al., Peter Lang, 2011, p. 53.

32. Rudolf Jelínek, «Offene Tribüne: Wien, gestern und heute», WuS, novembre 1963, p. 9. 
Wir sind überzeugt davon, daß unser „kapitalistisches System“ besser ist als das kommunistische [...] drüben. Wir sind überzeugt davon, daß es sich auch in Zukunft erweisen wird. Ich für meinen Teil aber bin auch überzeugt davon, daß man der Sache des Westens keinen guten Dienst erweist, wenn man Behauptungen aufstellt, die nicht zu beweisen, die unwahr sind.

Nehmen wir die Menschenschlangen vor den Geschäften. „Die da drüben hungern", behaupten manche, die ihrer ansichtig geworden sind. [...] Ich möchte betonen, daß ich kein Wirtschaftsfachmann bin und ich glaube auch, daß ein Körnchen Wahrheit drin ist, wenn man die Schlangen vor den Geschäften mit der Planwirtschaft in Zusammenhang bringt. Aber: es ist ein Unsinn zu behaupten, die haben nichts zu essen, die haben nichts anzuziehen, die verhungern. [...]

Daß die Kirchen leer sind, daß sich keiner getraut, ein Gotteshaus aufzusuchen, ist ebenfalls einer jener unvorsichtigen Behauptungen, die immer wieder aufgestellt werden. [...] Wir konnten an Sonntagen feststellen, daß die Gotteshäuser voll waren ${ }^{33}$...

Concernant le deuxième objectif de la revue - l'amorce de relations culturelles -, WuS déploie un arsenal de thématiques visant à mettre en avant les convergences nombreuses entre les deux pays. On peut classer ces thématiques en trois grandes catégories. La première s'articule autour des notions de voisinage et de frontière ${ }^{34}$. L'une des particularités de la relation austro-tchécoslovaque réside en effet dans la proximité géographique, compliquée toutefois par le fait que les deux États se trouvent à la jonction de deux blocs antagonistes. Même si l'Autriche est théoriquement neutre, obligation à laquelle $W u S$ ne manque pas de la rappeler régulièrement, souvent avec ironie, pour dénoncer les violations de cette neutralités ${ }^{35}$, il n’en reste pas moins que la Tchécoslovaquie

33. Justus Witt, «Besuch drüben», WuS, juillet 1965, p. 12-13.

34. Sur cette notion, voir notamment Andrea Komlosy, Václav Bůžek et František Svátek (dir.), Kulturen an der Grenze? Waldviertel. Weinviertel. Südböhmen. Südmähren, Wien, Promedia, 1995.

35. Les motifs de ces accusations sont divers : reproche fait à l'Autriche d'accueillir d'anciens nazis sur son territoire ou d'autoriser la tenue de rassemblements d'Allemands des Sudètes, ou encore de se tourner vers la CEE. On trouve ainsi une double allusion sarcastique à l'«Autriche neutre» dans l'article «Eingetragen unter Nr. 110 in der Kriegsverbrecherliste 44: Ferdinand Durčanský», WuS, janvier 1963, p. 12-13. WuS adresse plusieurs avertissements à l'Autriche, suspectée de ne pas respecter son engagement de neutralité : «Unser Nachbar Österreich. Ein Beitrag von Dr. h. c. Josef Plojhar, Minister für Gesundheitswesen», WuS, février 1962, p. 9 ; "Offene Tribüne: In Neutralität verhungern? Dr. Helene Legradi, Wien», WuS, juin 1963, p. 9, qui proteste contre l'adhésion de l'Autriche à la CEE ; «ČSSR-Österreich. Zum Jahrestag (Interview von Otto Klička)», WuS, mai 1963, p. 12-13 ; "Großdeutsche Gymnastik», WuS, septembre 1966, p. 12-13, reprochant à l'Autriche d'avoir autorisé à Innsbruck un rassemblement nazi «sous couvert de la neutralité autrichienne» (unter dem Deckmantel der österreichischen Neutralität) ; «Zum Jahresende», WuS, décembre 1967, p. 12-13 ; 
se trouve de l'autre côté du rideau de fer et que le traitement récurrent du motif de la frontière dans la revue révèle de la part de Prague une résistance à la perspective dêtre laissé de l'autre côtés ${ }^{36}$. Le nombre accru - en particulier à partir de 1963-1964 - d’articles consacrés au raccourcissement de la distance séparant les deux États du fait d'une part de l'assouplissement de la législation sur les flux touristiques et d'autre part des innovations et progrès techniques dans le domaine des transports montre bien la volonté de la Tchécoslovaquie de s'arrimer à l'Autriche ${ }^{37}$. Celle-ci est appelée, en vertu même de sa neutralité, à souvrir à ses voisins est-européens et à leur faire une place :

Könnte Wien, die Hauptstadt eines neutralen Landes, seine Kulturstätten nicht öfter und reichlicher auch dem Osten öffnen? Eine Kulturtribüne für die ganze Welt schaffen? Hier könnten westliche und östliche Künstler nicht auf Konferenzen, sondern vor allem bei ihrem künstlerischen Auftreten zusammenkommen. Das würde nicht nur dem gegenseitigen Kennenlernen und Näherkommen dienen, sondern auch das Wiener Kulturleben modernisieren und seinen Platz in der Weltkultur festigen ${ }^{38}$.

La deuxième grande thématique visant à souligner les convergences consiste en la réaffirmation constante du rôle médiateur des arts et de la culture, incarné en particulier par des écrivains et artistes capables, $\mathrm{du}$ fait de leur origine et de leur parcours, de faire le trait d'union entre culture autrichienne et culture tchécoslovaque :

An den kulturellen Verbindungen zwischen Österreich und den böhmischen Ländern, die in den Beziehungen der beiden Völker seitjeher die beste Vermittlerrolle spielen, haben Künstler einen bedeutenden Anteil. Im 19. Jahrhundert, in der franzisko-josephinischen Ära, da die Donaumonarchie von nationalen Kämpfen erschüttert wird, sind es die großen Dichter, die in den politischen Wirrnissen der menschlichen Verständigung ihre Stimme geben, besonders jene, denen beide Welten, die deutsche wie die slawische, gleich vertraut sind ${ }^{39}$.

J. Kružík, «Durchaus unneutraler Heimattag», WuS, novembre-décembre 1969, p. 18-19.

36. Pas moins de 7 articles entre 1962 et 1969 annoncent dès le titre le traitement de cette thématique.

37. À titre d’exemples : «Eine schöne Höllenfahrt», WuS, mars 1964, p. 18-19; «Die Strecke Wien-Prag ist kürzer geworden» et «Rakete Wien-Bratislava», WuS, septembre 1964, p. 12-13 et 17 ; «Flieg mit uns», WuS, juin 1966, p. 16.

38. "Gast der Wiener Woche war der Filmkritiker F. Goldscheider aus Prag», WuS, février 1963, p. 20-21.

39. Alois Hofman, «Mittlerin aus Mähren», WuS, mai 1966, p. 22-23. Alois Hofman est par ailleurs l'auteur d'une étude pionnière sur la revue bohémiste Ost und West : Die Prager Zeitschrift „Ost und West“. Ein Beitrag zur Geschichte der deutsch-slawischen Verständigung im Vormärz, Berlin, Akademie-Verlag, 1957. 
Le premier de ces passeurs est Adalbert Stifter (1805-1868) à propos duquel on peut lire, en préambule d'un article consacré au roman Witiko: "Gibt es ein stärkeres literarisches Bindeglied zwischen Österreich und Böhmen als Adalbert Stifter ${ }^{40}$ ?» Il faut également citer le compositeur Gustav Mahler ${ }^{41}$, l'architecte Adolf $\operatorname{Loos}^{42}$, les écrivains Ferdinand von Saar ${ }^{43}$, Maria Ebner-Eschenbach ${ }^{44}$, Franz Grillparzer, ou encore le dramaturge autrichien Franz Theodor Csokor (1885-1969) ${ }^{45}$.

Dans la troisième et dernière catégorie de thématiques destinées à souligner la proximité entre les deux pays s'inscrit la comparaison quasi systématique entre Vienne et Prague. Qu'il s'agisse de membres de la rédaction en reportage à Vienne, d'artistes en tournée à Prague ou à Vienne, ou de simples touristes, tous font valoir à l'envi l'étonnante similitude entre les deux capitales, qui concerne à la fois l'architecture urbaine, les traditions musicales, ou encore le goût de leurs habitants pour la bonne chère, et il n'est pas anodin que ce discours soit porté à la fois par des Tchèques en visite à Vienne et des Autrichiens en visite

40. Leo Brod, «Prag gewidmet: Adalbert Stifters Witiko», WuS, février 1965, p. 12. Un article consacré au photographe Karel Plicka («Die Moldau Karel Plickas und Adalbert Stifters. Der Altmeister der tschechischen Photographie stellte in Wien aus», WuS, août 1963, p. 20-23) soulignait déjà : "Wie sehr das Werk des Böhmenwalddichters zum gemeinsamen Kulturgut der böhmischen und der österreichischen Länder gehört, soll in dieser Zeitschrift ein andermal aufgezeigt werden...» L'article "Anerkennung aus Linz für die Stiftergedenkstätte in Oberplan» d'Aldemar Schiffkorn (WuS, juin 1965, p. 12-13) développe une nouvelle fois cette métaphore du trait d'union : «An der Seewand des Plöckensteinsees steht das vom österreichischen Architekten Heinrich von Ferstel entworfene Monument - der weithin sichtbare Obelisk. Und über dem Grab des Dichters in Linz erhebt sich der Grabstein seines aus Böhmen kommenden Landsmannes Johannes Rint. Liegt darin nicht eine Metapher umschrieben, die Stifters Werk kennzeichnet - Böhmen und Österreich?»

41. Le musicologue et collaborateur de Im Herzen Europas Pavel Eckstein (1911-2000) examine les liens de Mahler avec la culture tchèque dans « Gustav Mahler stammte aus... In der Tschechoslowakei und in Wien auf Mahlers Spuren», WuS, avril 1963, p. 20-21.

42. Otakar Nový, «Adolf Loos. Der Menschen menschlichster», WuS, avril 1965, p. 21-23. Sur les liens de Loos avec la culture tchèque, voir l'article de Cécile Poulot dans ce numéro d'Austriaca. WuS semble ainsi précurseur de la redécouverte de l'héritage loosien par la Tchécoslovaquie.

43. Leo Brod, «Eine Prager Inspiration Ferdinands von Saar», WuS, janvier 1966, p. 21. L'auteur conclut son article en ces termes : «Sein hinterlassenes Werk ist ein geistiges Band zwischen den böhmischen Ländern und Wien.»

44. Alois Hofman, «Mittlerin aus Mähren», art. cité.

45. "Zeuge einer Zeit in Prag», WuS, juillet 1967, p. 12-13. L'interview se termine par ce vœu de Csokor : «Denn ich bin der absoluten Anschauung, daß Österreich kein Brückenkopf $z u$ sein hat, sondern eine Brücke, und daß der Friede für die Zukunft von jenem Punkt ausgehen möge, von dem die beiden schrecklichen Kriege der letzten Zeit ausgegangen sind: vom Herzen Europas - eben meiner österreichischen Heimat.» 
à Prague. Le critique de cinéma tchèque František Goldscheider ne se lasse pas d'énumérer ces points communs :

Prag und Wien haben in der Tat vieles gemeinsam. Zum Beispiel die Gassen. Die lieben, alten Gassen, die durch ein Labyrinth von Windungen und Durchhäusern ins Unbekannte führen. [...] Die Wiener und Prager haben noch eine gemeinsame Leidenschaft. Den ständigen inneren Kampf zwischen dem naschhaften Gaumen und dem Bewußtsein, daß allzuviel ungesund ist. [...] Zweifellos gibt es noch viel Gemeinsames. Z. B. die Verwandten, die böhmische Tante und die österreichische Großmama, ein Stück Geschichte (wenn wir sie auch von verschiedenen Standpunkten aus betrachten) und die Hinterlassenschaft Kaiser Franz Josefs ${ }^{46} \ldots$

Le journaliste et photographe Milan Škarýd, qui inaugure en 1965 une série d'articles intitulés «Spiegelbild beider Metropolen» comparant les deux capitales ${ }^{47}$, reprend lui aussi ce topos: «weil Wien Prag so ähnlich ist» (notons que c'est Vienne qui ressemble à Prague, et non l'inverse); «daß sich halb Wien ganz gut tschechisch verständigen könnte, was in unserem Unterbewußtsein das Gefühl hervorrief, daß wir hier irgendwie zu Hause sind ${ }^{48} »$. Côté autrichien, on trouverait la même emphase, à en croire l'auteur du témoignage «Besuch drüben»:

Man hat sofort das Gefühl, man ist daheim. Es sind dieselben Gesichter, sie sprechen ähnlich wie wir, vor allem ist das „Böhmakeln" unserem Ohr ein gewohnter Klang. Wer kennt nicht in seiner näheren Umgebung einen Schuster oder Schneider, der nicht nur dem Namen, sondern auch der Sprache nach hüben wie drüben leben könnte ${ }^{49}$ ?

Ou celui de l'intendant général du festival «Wiener Festwochen», plaidant pour un espace culturel commun au centre de l'Europe :

Wenn wir davon sprechen, daß unsere Staaten die Mitte Europas sind und wenn wir diese Mitte als Kulturraum verstehen, können wir seine historisch-kulturellen Grundlagen nicht übersehen, ohne Rücksicht auf die heutige Verschiedenheit der politischen Regime. Wenn wir alle übereinstimmend diesen Kulturgedanken bejahen und wenn wir tatsächlich diese "Mitte Europas" sind, warum sollten wir dann nicht gemeinsam die kulturelle Stärke dieses mitteleuropäischen Raums manifestieren [...]? Daß man die gemeinsame Vergangenheit nicht verleugnen kann, das sehen wir am besten bei uns beinahe in jeder Familie. [...] wenn wir unsere gutnachbarlichen Beziehungen auf wirtschaftlichem Gebiet ständig

46. «Gast der Wiener Woche war der Filmkritiker F. Goldscheider aus Prag», WuS, février 1963, p. 20-21.

47. 14 articles d'octobre 1965 à décembre 1966.

48. «Wiener Streiflichter», WuS, février 1964, p. 22-24.

49. Justus Witt, «Besuch drüben», WuS, juillet 1965, p. 12-13. 
vertiefen, dann muß es notwendigerweise auch auf dem Gebiet der kulturellen Beziehungen geschehen ${ }^{50}$.

Cette recherche de conciliation, ces plaidoyers pour un rapprochement par le biais de la culture ne sauraient toutefois escamoter des sujets de friction rémanents. La question de la neutralité autrichienne en est un premier, nous l'avons évoqué. L'héritage habsbourgeois, encore largement associé à la notion de "ténèbres» (Temno, en tchèque), en constitue un deuxième. Ce thème, fondé sur le mythe de l'obscurité qui se serait abattue sur les Pays tchèques après la défaite de la Montagne blanche en $1620^{51}$, était déjà très présent dans la revue Im Herzen Europas avant le lancement de $W u S$; il l'est un peu moins dans la version autrichienne de la revue et on peut même lire en 1969 un article de Václav Holzknecht, président de la Société tchécoslovaco-autrichienne, rappelant le potentiel du passé habsbourgeois comme facteur de rapprochement entre les deux pays ${ }^{52}$. Cette référence fréquente au Temno et ces descriptions très négatives du passé habsbourgeois ont sans doute quelque chose à voir aussi avec les craintes tchécoslovaques d'une possible restauration de la monarchie Habsburg autour d'Otto Habsburg auquel l'Autriche a accordé un passeport en $1966^{53}$. Le troisième point de conflit est lié au passé national-socialiste : $W u S$ dénonce régulièrement la complaisance de l'État autrichien vis-à-vis d'anciens criminels nazis et des lobbys sudètes, accusés de relayer une idéologie proche de celle du nazisme. Il faut toutefois souligner que cet aspect est largement

50. «Nachbarn an der Donau» (Interview avec Dr. Ulrich Baumgartner), WuS, juin 1967, p. $10-11$.

51. Sur ce mythe, voir notamment : Josef Petraň, «Le mythe de la Montagne blanche», Marie-Elizabeth Ducreux (trad.), dans Marie-Elizabeth Ducreux et Antoine Marès (dir.), Enjeux de l'histoire en Europe centrale, Paris, L'Harmattan, 2002, p. 15-49, et Hélène Leclerc, Une littérature entre deux peuples. Écrivains de langue allemande en Bohême 1815-1848, Toulouse, PUM, 2011, p. 191-209.

52. Václav Holzknecht, «Blick über die Grenze: Gute Nachbarn sein», WuS, février 1969, p. 27.

53. Paul Ullmann indique que l'émissaire tchécoslovaque à Vienne rapporte ce fait le 29 juin 1966 en mentionnant son inquiétude que l'extrême droite autrichienne puisse se rassembler dorénavant autour de la personne d'Otto Habsburg (Eine schwierige Nachbarschaft, op. cit., p. 149). Les Habsbourg étaient interdits d'entrée sur le territoire autrichien depuis la loi du 3 avril 1919 qui avait déchu la maison de Habsbourg-Lorraine de tous ses droits et confisqué ses biens au profit de la République ; cette loi avait été promulguée en même temps que la loi d’abolition de la noblesse (Adelsaufhebungsgesetz) qui proscrivait entre autres le recours à la particule nobiliaire. La peur d'une restauration de la monarchie nétait pas nouvelle, elle caractérisa la Tchécoslovaquie dès sa fondation, et en particulier Edvard Beneš. 
plus développé dans la version de la revue destinée à la République fédérale, l'Autriche pouvant même apparaître comme une victime du militarisme allemand au même titre que la Tchécoslovaquie ${ }^{54}$.

En dépit de la volonté de mettre en avant des convergences, les difficultés demeurent, comme l'atteste l'interview d'Otto Klička en décembre $1967^{55}$. Il s'agit d'initiatives émanant de la Tchécoslovaquie ; Vienne se montra longtemps frileuse vis-à-vis de l'institutionnalisation de relations culturelles avec Prague. La revue WuS apparaît comme un instrument de public diplomacy visant à mobiliser l'opinion autrichienne, à lutter contre la prétendue «désinformation», pour employer un terme plus contemporain, ou la mésinformation. On peut s'interroger sur l'intérêt de cette offensive culturelle vers l'Autriche. En tant que pays neutre, l'Autriche semblait peut-être plus perméable aux influences tchécoslovaques, plus susceptible de souvrir à sa culture, et une fois ce rapprochement opéré, la Tchécoslovaquie aurait disposé d'un voisin amical lui permettant d'aborder plus sereinement sa relation à l'Allemagne. Face aux maigres succès diplomatiques de la Tchécoslovaquie vis-à-vis de la RFA, Prague tente de miser sur la carte autrichienne. Car on ne saurait perdre de vue que la préoccupation de la politique étrangère tchécoslovaque demeurait la question allemande, comme le montre le fait que la conclusion de l'accord sur les biens autrichiens (1974) n'intervint qu'après la signature du traité de Prague avec la RFA (1973). On constate un évitement manifeste des sujets qui fâchent vraiment (la question des biens autrichiens nest jamais abordée dans la revue) ; tout au plus admoneste-t-on gentiment l'Autriche sur ses prétendus manquements à la neutralité et on se tait sur son passé nationalsocialiste et les échecs de la dénazification. L'objectif est et demeure celui de la conciliation et du rapprochement.

54. Interview d’Antonín Šnejdárek par Gustav Solar : «Tschechoslowakisch-österreichische Historikerkontakte», WuS, mai 1964, p. 16.

55. O. Klička, «Zum Jahresende», WuS, décembre 1967, p. 12-13. 